\title{
Validity Of Congo Red Agar And Modified Congo Red Agar To Detect Biofilm Of Enterococcus Faecalis
}

\author{
Rina Normanita ${ }^{1,2}$, Kuntaman Kuntaman ${ }^{1,2}$, Eddy Bagus Wasito ${ }^{1,2}$ \\ ${ }^{1}$ Department of Clinical Microbiology Faculty of Medicine Universitas Airlangga \\ ${ }^{2}$ Dr. Soetomo Hospital Surabaya J1. Mayjen. Prof. Dr. Moestopo No. 6-8, Surabaya, Jawa \\ Timur 60286 Phone : (+62 31) 5501078 \\ Email : bangunan2211@gmail.com \\ Receive : Jan $15^{\text {th }}$ 2020. Revised : May $20^{\text {th }}$ 2020. Published: June $12^{\text {th }} 2020$ \\ DOI: https://doi.org/10.22219/sm.Vol16.SMUMM1.11064
}

\begin{abstract}
Purpose: Enterococcus faecalis causes nosocomial infections such as bacteremia, urinary tract infections, intraabdominal infections, and endocarditis. These infection is associated with biofilm and intrinsically resistant to many antibiotics. This study aims to determine the validity of the CRA and MCRA for detecting biofilms of Enterococcus faecalis

Method: This is a laboratory observational study with 30 sample of Enterococcus faecalis. We performed biofilm examination for Enterococcus faecalis by using Congo red Agar, Modified Congo red Agar and Microtitter Plate Assay as gold standard.

Result: Both MCRA and CRA were compared MPA as a gold standard was obtained $p$ value is 0.309 ( $p>$ $0.05)$, with a Kappa agreement coefficient is 0.067 , which indicates there is no significant agreement to detect biofilm of Enterococcus faecalis. MCRA and CRA have almost no compatibility with MPA for biofilm forming of Enterococcus faecalis.

Conclusion: Both MCRA and CRA has a very high sensitivity (100\%), but the specificity is very low $6.67 \%$ for detecting the biofilms of Enterococcus faecalis. MCRA and CRA can not determine negativity well and it have a high false positive rate, so to increase specificity of biofilm forming, we must combine these method with the others.
\end{abstract}

Keywords: Biofilm, Enterococcus faecalis, CRA, MCRA, MPA

Copyright (C) 2020, First Author et al This is an open access article under the CC-BY-SA license

\section{INTRODUCTION}

The infections that caused by biofilm-producing bacteria has become an urgent in clinical setting. Biofilms producing bacteria is associated with a number of persistent infections that respond very poorly to antibiotic therapy, change ability of the bacteria to survive against immune responses and help spreading of antibiotic resistant nosocomial infection (Gaca et al., 2012, Ponnusamy and Nagappan, 2013). One of the agents that commonly causes of severe nosocomial 
infections, such as bacteremia, urinary tract infections, intra-abdominal infections, and endocarditis, is Enterococcus faecalis (Richard et al, 2000).

The ability of Enterococcus faecalis forming biofilms shows their virulence. This ability enables colonization of inert and biological surfaces while protect itself against antibiotic substances and mediate adhesion to host cells (Hashem et al, 2017). The structure of biofilms provides an optimal micro-environment for growth and facilitates the transmission of cellular genetic elements between bacteria (Sienko et al, 2015). Biofilms play an important role in bacterial virulence, because they protect bacteria, especially uro-pathogen from the antibactericidal activity of antibiotic in various ways. This bacterial defense mechanism is due to the changing the of bacterial cells characteristics in environmental adaptations, such as slowing growth of microorganisms or undergoing metabolic dormant, which leads to increase antibiotics tolerance and resistance (Fujiwara et al., 1998; Donlan, 2003; Trautner et al., 2004).

Strains of bacteria that produce biofilms, including Enterococcus faecalis, show higher resistance to antibiotics compared to those that do not produce biofilms. Levy's (2002) shows that $100 \%$ of enterococci strains produce biofilms that are resistant to 2 (two) or more antibiotics and even to the phenotypic MDR. Komiyama et al (2017) said that Enterococcus faecalis often forms biofilms in stents and other devices, which require long-term antibiotic administration when removal of the devices are not possible. Chen and Wen (2011) and Atray and Atray (2015) show that biofilm production is often associated with organisms that are in the urinary tract for longtime and dramatically increases antibiotic resistance.

Ch'ng et al. (2018) revealed that Enterococci cause 25\% of all catheter-related urinary tract infections. These bacteria are also often isolated in wounds and are increasingly found in infective endocarditis. All of these infections are associated with biofilms. Enterococcal biofilms are intrinsically resistant to antibiotics so it pose a serious obstacle in the treatment of infections.

Christopher (2003), revealed that Enterococcus faecalis can form biofilms in vitro, so these organisms are often isolated from biofilms on the surface of various medical devices. However, the molecular mechanisms that regulate biofilm formation in clinical isolates are largely unknown. The formation of biofilms as pathogenesis of disease can be detected using several methods, including: Microtiter Plate Assay (MPA), Congo Red Agar (CRA) and Tube Method (TM) (Hasan et al., 2011). The Microtiter Plate Assay (MPA) method was considered as the gold standard for biofilm examinations (Christensen et al., 1985).

According to Kaiser et al. (2012), the CRA is considered to have several advantages over other methods, cheap, fast, and easy to do even for small laboratories, and does not require technical expertise. However, this method also has disadvantages because it needs subjective evaluation. Triveda et al (2016) considered that sensitivity and specificity of the CRA to detect biofilm Enterococcus were 25\% and 46.67\% respectively. Melo et al (2013), considered that the sensitivity and specificity of the MCRA method using additions of glucose to detect the biofilms production of Staphylococcus aureus were $90.63 \%$ and $90.6 \%$ (compared with MPA) as the gold 
standard). The sensitivity and specificity of the MCRA (compared with PCR) were $89 \%$ and $100 \%$. There has been no research comparing the sensitivity and specificity of CRA to compare with MCRA to detect biofilms producing Enterococcus faecalis. This study aims to determine the sensitivity and specificity of CRA and MCRA for detecting biofilms producing Enterococcus faecalis from clinical isolates at Dr. Soetomo Hospital, Surabaya.

\section{MATERIALS AND METHODS}

The observational analytic study using the clinical isolate of Enterococcus faecalis was conducted at Clinical Microbiology Department, Dr. Soetomo Hospital Surabaya. A total of 30 sample were collected by consecutive sampling from June - August 2019. The inclusion criteria is the Enterococcus faecalis that was identified based on the automatic identification test of the BD PhoenixTM Automated Microbiology System and / or Vitek 2 Compact System.

Isolates were obtained from culture specimens of urine, blood and sterile fluids, sputum, and pus. Bacterial isolates were stored into cryotubes containing liquid media of tripticase soy broth and $15 \%$ glycerol in a deep freeze at temperature minus $80^{\circ} \mathrm{C}$.

Procedure for examining the biofilm production of Enterococcus faecalis used Congo Red Agar (CRA), Modified Congo Red Agar (MCRA) and Microtiter Plate Assay (MPA) as a gold standard. Isolates stored at $-80 \mathrm{oC}$ using Tryptone Soy Broth (TSB) media were sub-cultured on Sheep Blood Agar (SBA) incubated for 24 hours at 370C. Some colonies with identical morphology were made bacterial suspensions according to standard 0.5 McFarland, using a Nephelometric device. Each microtiter plate flat-bottomed 96 well polystyrene were filled with $180 \mu \mathrm{l}$ TSB. Next $20 \mu \mathrm{l}$ of bacterial suspension was added to the well, the plate was closed and incubated at 370C for 24 hours. After then the bacterial suspension in plate well was removed then washed with $300 \mu \mathrm{l}$ phosphate buffer saline $(\mathrm{pH}$ 7.2) three times and dried in an upside down position. Biofilms are formed by bacteria bound to wells, fixed with $150 \mu \mathrm{l}$ methanol in each well and allowed to stand for 20 minutes. The microtiter was dried by tapping and then left in an upside down position and then stained with crystal violet $(0.1 \%)$ for five minutes, the excess stain was washed with deionized water and the plates was dried. Optical density (OD) of bacteria bound by staining is distinguished by ELISA autoreader with a wave length of $595 \mathrm{~nm}$ (OD 595nm) (Shridhar, 2019). Biofilm (+) in MPA showed optical density between 0.066 - 0.132 for moderate and upper 0.132 for strong, while biofilm (-) in MPA showed optical density between 0.033 and below 0.066 (Figure 1) 


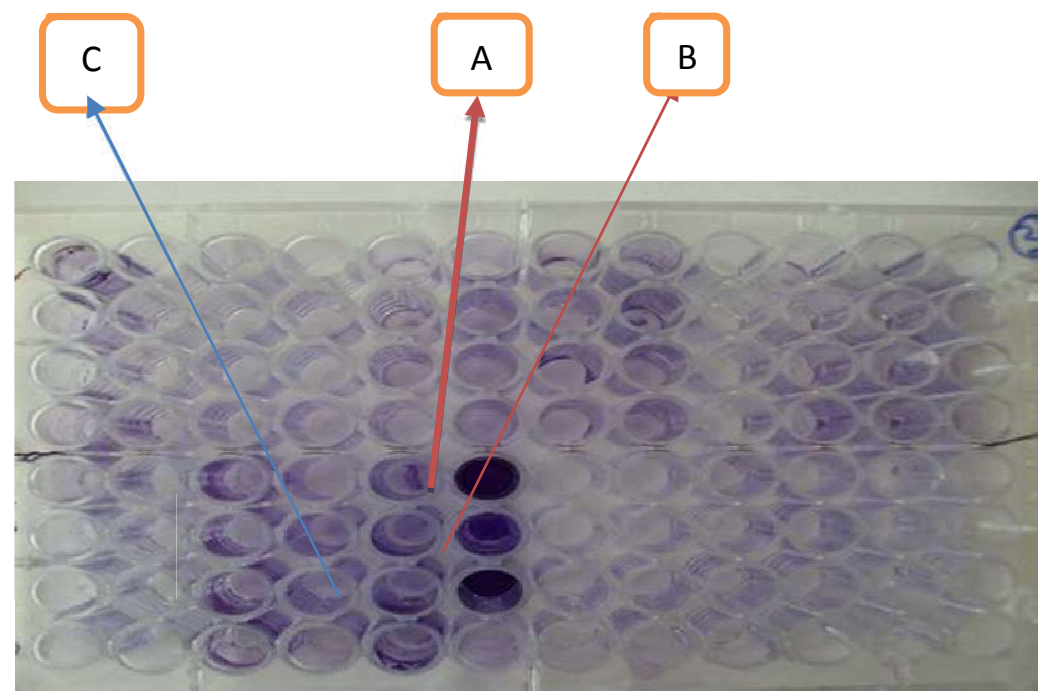

Figure 1. Microtiter Plate Methode (MPA) : A: strong positive, B: moderate positive, C: Negative (MPA on the ELISA reader show the presence of biofilms at $595 \mathrm{~nm}$ )

Congo Red Agar (CRA) is conducted by streaking, while Modified Congo Red Agar (MCRA) is conducted by spot inoculation technique. Observations were made by assessing the colors exhibited by bacterial colonies growth on the surface of the Congo Red Agar. (Figure 2) Black and brown colonies indicate that bacteria produced biofilms, red colonies indicate bacteria did not produce biofilms

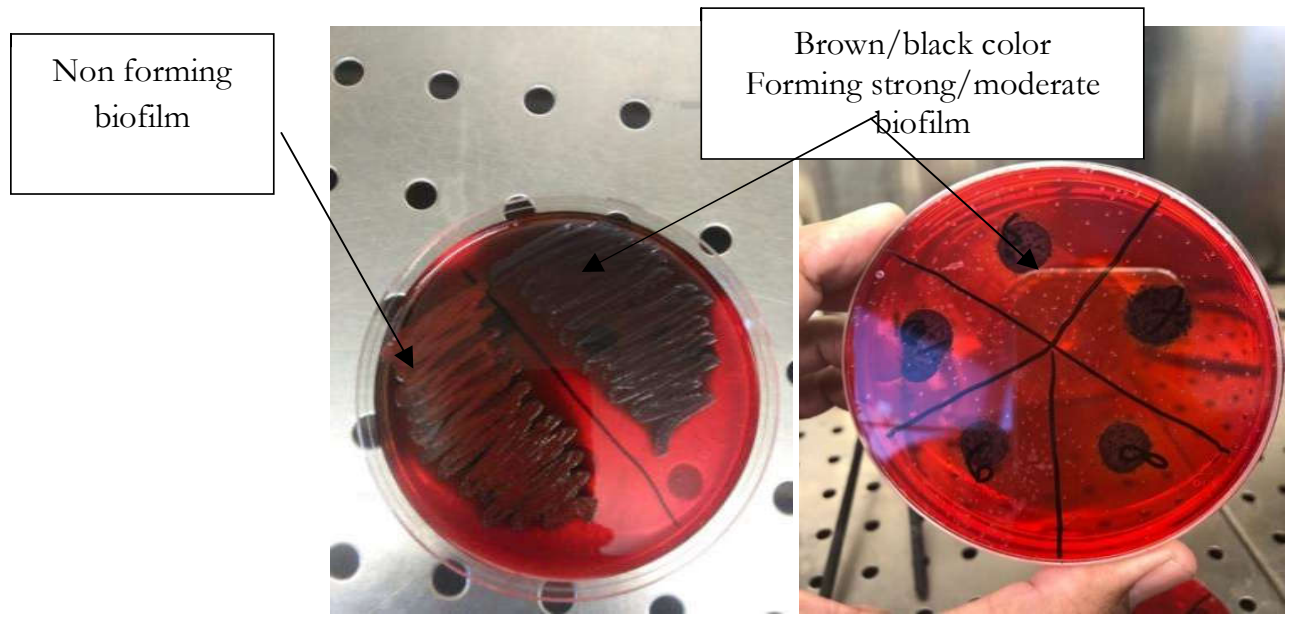

Figure 2. Biofilm formation test for CRA and MCRA methods

Analysis of data was presented cross-tabulation and assessed the sensitivity and specificity for CRA and MCRA. The data was analysed with the SPSS 17.0 software

\section{RESULTS \& DISCUSSION}

Total 30 specimens were examined. Most of the number of patients were dominated by male, which there are 17(56.7\%) male and $13(43.3 \%)$ female. By age group (1 month - 85 years), 
with an average age of 43.8 years. The most specimens of Enterococcus faecalis from urine were 18 isolates $(60 \%)$, followed blood were 11 isolates $(36.7 \%)$, pus was 1 isolate $(3.3 \%)$ (Table 1 )

Table 1. Characteristics of specimens of E. faecalis

\begin{tabular}{|c|c|c|c|}
\hline Variables & & Frequency & $\%$ \\
\hline \multirow[t]{3}{*}{ Specimens } & Urine & 18 & 60 \\
\hline & Blood & 11 & 36,7 \\
\hline & Pus & 1 & 3,3 \\
\hline \multirow[t]{2}{*}{ Sex } & Male & 17 & 56.7 \\
\hline & Female & 13 & 43.3 \\
\hline \multirow[t]{7}{*}{ Clinical Diagnostic } & UTI & 10 & 33.3 \\
\hline & Pneumonia / LRTI & 7 & 23.3 \\
\hline & Abdominal Infection & 3 & 10 \\
\hline & Burn & 2 & 6.7 \\
\hline & Fracture & 4 & 3.3 \\
\hline & Abcess & 1 & 3.3 \\
\hline & Sepsis & 6 & 20 \\
\hline \multirow[t]{2}{*}{$\begin{array}{l}\text { Biofilm forming of } \\
\text { isolates }\end{array}$} & Positive & 29 & 96.7 \\
\hline & Negative & 1 & 3.3 \\
\hline \multirow[t]{2}{*}{ CRA } & Biofilm (+) & 29 & 96.7 \\
\hline & Biofilm (-) & 1 & 3.3 \\
\hline \multirow[t]{2}{*}{ MCRA } & Biofilm (+) & 29 & 96.7 \\
\hline & Biofilm (-) & 1 & 3.3 \\
\hline \multirow[t]{3}{*}{ MPA } & Biofilm (+) & 15 & 50 \\
\hline & Biofilm (-) & 15 & 50 \\
\hline & Total & 30 & 100 \\
\hline
\end{tabular}

Note : UTI = Urinary Tract Infection; LRTI = Lower Respiratory Tract Infection

The most diagnoses were Urinary Tractus Infection (UTI) of 10 isolates (33.3\%), pneumonia and acute lower respiratory infection of 7 isolates $(23.3 \%)$ and sepsis of 5 isolates 
$(16.7 \%)$ (Table 1). The result of biofilm examination showed that were positif in 29 (96.7\%) on CRA, $29(96.7 \%)$ of MCRA and $15(50 \%)$ in MPA. Table 2 . Biofilm (+) in CRA and MCRA showed black and brown colonies while biofilm (-) showed red colonies. Biofilm (+) in MPA showed optical density between 0.066 - 0.132 for moderate and upper 0.132 for strong, while biofilm (-) in MPA showed optical density between 0.033 and below 0.066 (Table 1).

There is no significant difference between CRA and MPA ( $p=0.309(p>0.05))$. Kappa agreement coefficient of 0.067 (away from 1) can be meant there is suitability between MPA and CRA to detect the biofilms formation in Enterococcus faecalis isolate is very low. Validity of CRA compared with MPA as gold standard is sensitivity of $100 \%$ and specificity of $6.67 \%$. The positive predictive value is $51.72 \%$ and negative prediction value is $100 \%$. The result showed that CRA method is not a good diagnosing tool to determine biofilm forming, because of very low specificity

Results of biofilms producing Enterococcus faecalis by CRA vs MPA is shown in cross tabulation (Table 7). That show CRA of positive and MPA of positive (forming biofilms) were 15 isolates $(50 \%)$, while CRA of negative and MPA of positive are $0(0 \%)$. The results of MPA of negative and CRA of positive of 14 isolates (46.7\%) while MPA and CRA that were respectively negative (not forming biofilms) were 1 isolate $(3.3 \%)$.

Table 2. Distribution of Biofilms Producing Enterococcus faecalis by CRA vs MPA

\begin{tabular}{cccc}
\hline & MPA Positive & MPA Negative & Total \\
\hline CRA Positive & $15(50 \%)$ & $14(46.7 \%)$ & $29(96.7 \%)$ \\
CRA Negative & $0(0 \%)$ & $1(3.3 \%)$ & $1(3.3 \%)$ \\
Total & $15(50 \%)$ & $15(50 \%)$ & $30(100 \%)$ \\
\hline
\end{tabular}

There is no significant difference between CRA and MPA ( $p=0.309(p>0.05))$. Kappa agreement coefficient of 0.067 (away from 1) can be meant there is suitability between MPA and CRA to detect the biofilms formation in Enterococcus faecalis isolate is very low. Validity of CRA compared with MPA as gold standard is sensitivity of $100 \%$ and specificity of $6.67 \%$. The positive predictive value is $51.72 \%$ and negative prediction value is $100 \%$.

Results of biofilms producing Enterococcus faecalis by MCRA vs MPA is shown in cross tabulation (Table 8). They shows that the Enterococcus faecalis isolates using MCRA and MPA were respectively positive (forming biofilms) were 15 isolates (50\%), while using MCRA of negative values and MPA of positive values there are $0(0 \%)$. The results of MPA of negative with MCRA of positive value of 14 isolates (46.7\%) while for MPA and MCRA of negative (not forming biofilms) were 1 isolate $(3.3 \%)$. The result showed that MCRA method is not a good diagnosing tool to determine biofilm forming, because of very low specificity. (Table 3) 
Table 3. Distribution of Biofilms Producing Enterococcus faecalis by MCRA vs MPA

\begin{tabular}{cccc}
\hline & MPA Positive & MPA Negative & Total \\
\hline MCRA Positive & $15(50 \%)$ & $14(46.7 \%)$ & $29(96.7 \%)$ \\
MCRA Negative & $0(0 \%)$ & $1(3.3 \%)$ & $1(3.3 \%)$ \\
Total & $15(50 \%)$ & $15(50 \%)$ & $30(100 \%)$ \\
\hline
\end{tabular}

There is no significant difference between MCRA and MPA ( $\mathrm{p}=0.309(\mathrm{p}>0.05))$. Kappa agreement coefficient of 0.067 (away from 1) can be meant there is suitability between MPA and MCRA to detect biofilms formation in Enterococcus faecalis isolate is very low. Validity of MCRA compared with MPA as gold standard is sensitivity of $100 \%$ and specificity of $6.67 \%$. The positive predictive value is $51.72 \%$ and negative prediction value is $100 \%$.

Enterococcus faecalis is a predominant pathogen in urinary tract infections after Escherichia coli $(25 \%)$, is $16 \%$. This pathogen is commonly found in the lower digestive tract and can enter the urethra through contaminated devices (Tenke et al. 2006). The high incidence of severe Enterococcus faecalis infection results in increased morbidity and mortality as well as hospital costs and length of stay (Linden, 2003) Detection of the formation of Enterococcus faecalis biofilms can be done by several methods including Microtiter Plate Assay (MPA), Congo red Agar(CRA) and Modified Congo red Agar (MCRA). The mechanism of colony color change in CRA media occurs because Congo red stain will interact with polysaccharides which are secondary metabolic products of bacteria and growth media so that they form complexes with stain that cause the colonies to appear dark in color (Jain and Agarwal, 2009). Bacteria fermented sugars or polysaccharides needed to produce certain metabolites, that combine with Congo red so as give a black colony color that indicates the formation of biofilms (Mack et al, 1992).

Phenotypic evaluation of CRA in order to show the formation of black color associated with the presence of sucrose in CRA media. The concentration of sucrose is contained in the medium affects the production of EPS. When the concentration of sucrose is low, the diffusion of black pigment from the colony is also reduced. Sucrose in CRA acts as a carbon source. As a comparison in this study using MCRA. According to Kaiser et al. (2012), MCRA to be a modification of the way of inoculation of Enterococcus faecalis so that the interpretation of colony and pigmentation is more easily evaluated. The difference in MCRA compared to CRA, is changing the streaking method to spot inoculation. MCRA method is considered to be more economical for the media used to identify biofilms. This method is fast, easy, sensitive, and reproducible and colonies growing on this medium can still be used for further analysis. The initial study proposed MCRA is an alternative MPA for screening biofilm forming (Knobloch, 2002) because it is easy to do, time-consuming, sensitive and specific, while MCRA and CRA have high sensitivity but very 
low specificity. MCRA and CRA can not determine negativity well and it will have a high false positive rate. So both of tool are good for catching actual case of Enterococcus faecalis but they also come with fairly high false positive rate. To increase specificity of detection of biofilm forming, we must combine MCRA or CRA method with the others. Actually we may use these method to detect biofilm forming, because MCRA and CRA have some advantages, i.e easy and simple implementation, cheap and simple production of media, and simple assessment of result.

MCRA and CRA is not fully able to show how the bacteria formed biofilm, because the mechanism of biofilm formation can go through several pathway. MCRA also has a difference compared to CRA, in which it is given glucose addition of 10g/l. Based on research Rossi et al (2016) showed an increase in the production of LPS in glucose that added media with levels of $0.08 \%, 0.15 \%$ and $0.2 \%$. Melo (2013) shows a positive correlation between an increasing of glucose concentration in the environment around bacteria and an increasing of biofilm formation.

The results is obtained the similarity of results between the CRA and MCRA methods because CRA and MCRA use sucrose and glucose which are polysaccharides to form biofilms while in MPA do not use glucose to form biofilm (Uphadayaya., 2010). Enterococcus faecalis has increased in producing biofilms if given glucose. Starvation is one of the most important factors that influences the formation of E. faecalis biofilms. When the alkalinity is higher, E. faecalis shows a reduced ability to form biofilms.. Some researchers have also shown that among E. faecalis, glucose supplementation increases biofilm formation. There is no difference between CRA and MCRA to detect rates of biofilms formation except in the case of Staphylococcal biofilms where MCRA is a better method than CRA for the same thing. (Panda, et al. 2016)

This study resulted several findings, are: (1) Urine is the most common specimen of Enterococcus faecalis isolate compared to other specimens; (2) Sensitivity and specificity of the CRA and MCRA is same to MPA so that CRA and MCRA can be used as an initial screening alternative to detect biofilm producing Enterococcus faecalis but cannot be used as a standardized microbiological examination as a determinant of biofilm formation; (3) MPA can be used as a detection tool for biofilm production in clinical isolates in Dr. Soetomo Hospital, Surabaya, given the high use of devices in hospitals. Dr. Soetomo Surabaya as the main referral hospital for East Indonesia

\section{CONCLUSION}

The conclusions that can be drawn from this study are: (1) MCRA have sensitivity of 100\% and specificity of $6.67 \%$ to detect biofilm producing Enterococcus faecalis compared with MPA as the gold standard in Dr. Soetomo Hospitals, Surabaya; (2) CRA have sensitivity of $100 \%$ and specificity of $6.67 \%$ to detect biofilm producing Enterococcus faecalis compared with MPA as the gold standard in Dr. Soetomo Hospitals, Surabaya; (3) MCRA and CRA can not determine 
negativity well and it will have a high false positive rate. To increase specificity of detection of biofilm forming, we must combine MCRA or CRA method with the others.

\section{LIMITATION}

We have some limitation in this study : sample size and limitation of financial resources and timing of study that might influence the interpretation of result

\section{ACKNOWLEDGMENT}

This research is fully supported by the Clinical Microbiology Unit of Dr. Soetomo Hospital Surabaya and the Institute of Tropical Diseases, University of Airlangga. Thank you, Prof. Kuntaman and Prof. Eddy Bagus Wasito as Advisor in this study. Thank you also the author goes to all staff analysts both at the Clinical Microbiology Unit of Dr. Soetomo Hospital Surabaya and the Institute for Tropical Diseases, University of Airlangga.

\section{REFERENCES}

Ch'ng, J., Chong, KLL.., Lam, LN., Wong, JJ., Kline, KA. (2018). Biofilm- Associated Infection by Enterococci. www.nature.com

Christensen GD., Simpson WA., Younge JA, (1985). Adherence Of Coagulase Negative Staphylococcus to Plastic Tissue Culture: Quantitative Model For The Adherence of Staphylococci to medical device. J Clin Microbiol 22: 996-1006

Christopher, J., Kristich, Li, Y., Cvitkovitch, DG., Dunny, GM. (2003). Esp-Independent Biofilm Formation by Enterococcus faecalis

Deepika, A.,Meena, A. (2015). Correlation between Biofilm Production and Antibiotic Resistance Pattern in Uropathogenic Escherichia coli in Tertiary Care Hospital in Southern Rajasthan, India. International Journal of Current Microbiology and Applied Sciences. 4:640-646

Donlan, RM., 2003. Biofilms: Microbial Life On Surface. Emer Infect Dis 8:881-890

Fujiwara T., Terao Y., Hoshino T., Kawabata S., Ooshima T., Sobue S. (1998). Molecular Analyses Of Glucosyltransferase Genes Among Strains Of Streptococcus Mutans. FEMS Microbiol Lett 161:331-336. CrossRef, Medline

Gaca, AO. (2013). Basal Levels of (p)ppGpp in Enterococcus faecalis: The Magic Beyond The Stringent Response. MBio 4, e00646-13 Genotype-Phenotype Correlations And Inhibition By Vancomycin. Sci Rep.7(1):5733

Hashem, YA.., Amin HM.., Essam TM.., Yassin,AS., Aziz RK.. (2017). Biofilm Formation In Enterococci: 
Hassan A., Usman J., Kaleem F., Omair M,. Khalid A., Iqbal M. (2011). Evaluation Of Different Detection Methods Of Biofilm Formation In The Clinical Isolate. Dilihat 29 April 2019. http://www.scielo.br/pdf/bjid/v15n4/v15n4a02.pdf

Jain A., Agarwal A. (2009). Biofilm Production, a Marker of Pathogenic Potential of Colonizing and Commensal Staphylococci. J Microbiol Methods 76: 88-92.

Kaiser, TDL. (2012). Modification Of The Congo Red Agar Method To Detect Biofilm Production

By Staphylococcus epidermidis. Diagnostic Microbiology and Infectious Disease 75:235-23

Knobloch, J., Horstkptte, MA., Rohde, H., Mack, D (2002). Evaluation Of Different Detection Methods Of Biofilm Formation in Staphylococcus aureus. Med Microbiol Immun 191: 1016.

Komiyama, EY., Lepesqueur, LSS., Yassuda, CG.,Samaranayake, LP., Parahitiyawa, NB., Balducci. (2017). Enterococcus spp in the Oral Cavity: Prevalence, Virulence Factors and Antimicrobial Susceptibility. PLoS ONE 11(9): e0163001. doi:10.1371/journal.pone.0163001

Linden, P. (2003). Can Enterococcal Infections Initiate Sepsis Syndrome? Current Infectious Disease Reports, 5:372-378

Mack, D., Siemssen, N., and Laufs, R. (1992). Parallel Induction By Glucose Of Adherence and A Polysaccharide Antigen Specific for Plasticadherent Staphylococcus epidermidis: Evidence For Functional Relation Tointercellular Adhesion. Infect Immun 60: 2048- 2057.

Melo, PC., Ferreirra, LM., Filhol, AN., Zafalon, LF., Vicente, HI., Souza, V. (2013). Comparison of Methods For The Detection Of Biofilm Formation By Staphylococcus aureus Isolated From Bovine Subclinical Mastitis. Brazilian Journal of Microbiology 44, 1, 119-124

Panda, PS., Chaudhary, U.,Dube, SK. (2016). Comparison of Four Different Methods for Detection of Biofilm Formation by Uropathogens. Indian Journal of Pathology and Microbiology. 59 (2):177-179

Ponnusamy, P., Nagappan, R. (2013.) Extended Spectrum Beta-Lactamase, Biofilm Producing Uropathogenic Pathogens And Their Antibiotic Susceptibility Patterns From Urinary Tract Infection- An Overview. Int. J.Microbiol. Res., 4(2): 101118

Richard, MJ., Edward, JR.., Culver, DH., Gaynes, RP. (2000). Nosocomial Infections In Combined Medical-Surgical Intensive Care Units In The United States. The National Nosocomial Infections Surveillance System

Shridirar, S., Dhanasree, B (2019). Antibiotic Susceptibility Pattern and Biofilm Formation in Clinical Isolate of Enterococcus spp. Interdisclipnary Perspectives on Infectious Disease. Dilihat 1 Mei 2019 .http : //www.hindawi.com/journals/ipid/2019/7854968

Sienko, A., Odjana, D., Majewsk, P., Sacha, P., Wieczorek, P., Tryniszewska, P. (2015).Comparison of Biofilm-Producing Enterococcus faecalis, Enterococcus faeceum And Unusual Enterococcus strain. European Journal of Biological Research; 7 (4): 291-298 
Stuart, LB. (2002). Factor Impacting On The Problem Of Antibiotic Resistance. Journal of Antimicrobial Chemotherapy . 49: 25-30

Tenke, P., Riedl, CR., Jones, GL., Williams, GJ., Stickler, D., Nagy, E. (2004). Bacterial Biofilm Formation on Urologic Devices and Heparin Coating as Preventive Strategy. Int J Antimicrob Agents. 23(1):67-74

Trautner, BW., Darouiche, RO. (2004). Role of Biofilm Infection In Urology. World JUrol 24:1320

Triveda, L., Gomathi, S. (2016). Detection of Biofilm Formation Among The Clinical Isolates Of Enterococci : An Evaluation Of Three Different Screening Methods. International Journal of Current Microbiology and Applied Science.

Upadhyaya, PM, Umapathy, BL., Ravikumar, KL. (2010) . Comparative Study for the Presence of Enterococcal Virulence Factors Gelatinase, Hemolysin and Biofilm Among Clinical and Commensal Isolates of Enterococcus faecalis. Journal of laboratory psychian. 